\title{
Akutdiagnostik bei Wirbelsäulenverletzungen
}

\author{
$\square$ Georg Böhmer, Hermann-Josef Böhm
}

\section{Zusammenfassung}

Die konventionelle Röntgendarstellung im antero-posterioren und seitlichen Strahlengang stellt unverändert die Basis der primären apparativen Untersuchung bei der akuten Wirbelsäulenverletzung dar. Selbstverständlich ist die vorherige Anamneseerhebung, die klinische und fachneurologische Untersuchung unerlässlich. In der weiterführenden Diagnostik stellt die Computertomographie, insbesondere in Spiral-CT-Technik, den Standard dar. Dies gilt sowohl für die Problemregionen der Wirbelsäule als auch für die Beurteilung des knöcher-

\section{Einleitung}

Der bewusstlose Unfallpatient gilt solange als Wirbelsäulenverletzter, bis das Gegenteil bewiesen ist.

Unverzichtbare Basis jeder Akutdiagnostik bei Wirbelsäulenverletzungen ist die Erhebung der Anamnese (Eigenoder Fremdanamnese), die klinische und fachneurologische Untersuchung. Die hieraus resultierenden Parameter sind beim bewusstseinsklaren Patienten einfach zu erheben und leiten bereits gezielt $\mathrm{zu}$ der verletzten Wirbelregion. Beim bewusstlosen oder polytraumatisierten Patienten besteht jedoch die Notwendigkeit, die Unversehrtheit der Wirbelsäule zu dokumentieren oder wie beim bewusstseinsklaren, die verletzte Region zu identifizieren. Dies bedeutet nach Stabilisierung der Vitalfunktionen und Ausschluss lebensbedrohender primär zu behandelnder Verletzungen die

OP-JOURNAL 2001; 17: 128-130

(C) Georg Thieme Verlag Stuttgart · New York

nen und ligamentären Verletzungsausmaßes. Weiterhin ist sie unverzichtbar bei der postoperativen Kontrolle des Repositionsergebnisses und der Implantatlage. Demgegenüber ist die kernspintomographische Untersuchung trotz ihrer Vorteile in der Kontrastauflösung der intraspinalen und diskoligamentären Weichteile beim akuten Wirbelsäulentrauma nur ein diagnostisches Verfahren der zweiten Wahl. Beim bewusstlosen, polytraumatisierten Patienten kann diese Untersuchung aufgrund der langen Untersuchungsdauer sogar kontraindiziert sein. säulenverletzung alle gewünschten Informationen zu liefern.

Das ideale alleinige bildgebende Verfahren in der Akutdiagnostik bei Wirbelsäulenverletzung gibt es nicht.

\section{Konventionelle Röntgenuntersuchung}

Die Standardröntgenaufnahme der Wirbelsäule im antero-posterioren und seitlichen Strahlengang ist der Grundpfeiler der Knochenabbildung (Abb.1a,b). Dies gilt für die herkömmlichen Filmfolien wie auch für die digital nachbearbeitbaren Speicherfolien. Röntgenaufnahmen sind schnell realisiert und haben die höchste räumliche Auflösung der radiologischen Methoden, jedoch ist ihre Kontrastauflösung beschränkt. Weichteile werden mit der Röntgenaufnahme nicht differenziert wiedergegeben. Standardröntgenaufnahmen zeigen immer eine Überlagerung anatomischer Strukturen, was die Interpretation erheblich erschwert [7].

Schichtaufnahmen, sensitiver als Standardröntgenaufnahmen in der Fraktur-

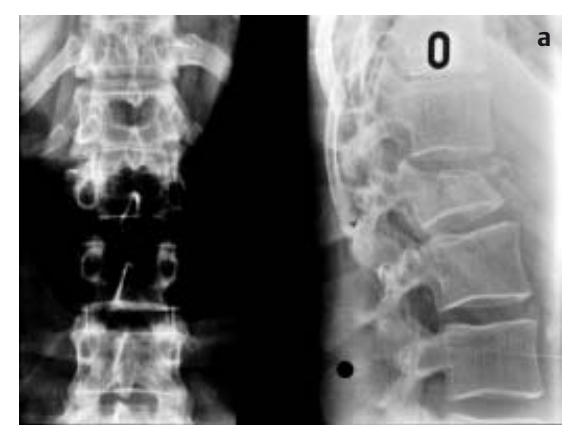

Abb.1 a, b Verlaufskontrolle einer instabilen LWK-2-Fraktur in der konventionellen Radiographie (a) sowie in seitlichen Zielaufnahmen prä- und postoperativ sowie nach Implantatentfernung (b).
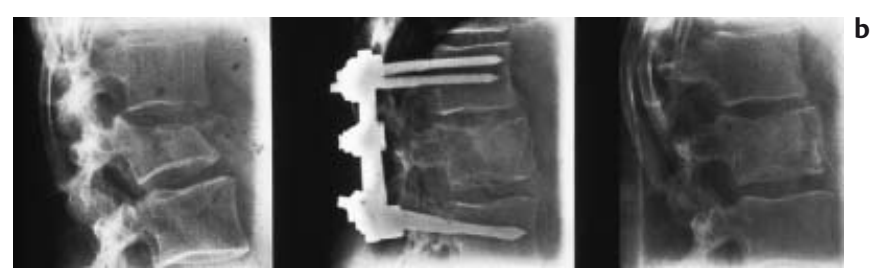

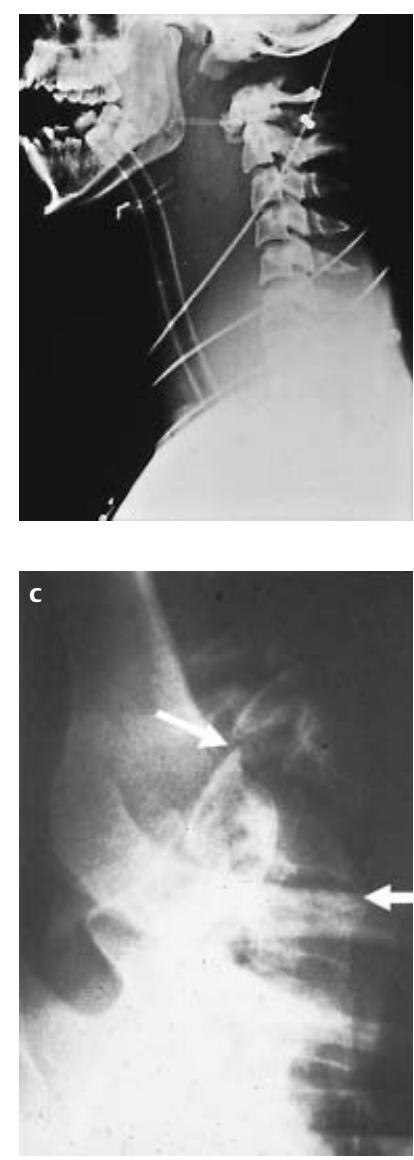

diagnostik, beinhalten die Problematik der kontrastarmen Darstellung und der fehlenden Weichteildarstellung und wurden deshalb abgesehen von wenigen Sonderindikationen, z. B. die Beurteilung der knöchernen Ausheilung einer Fraktur im oberen Halswirbelsäulenbereich [5], durch die Computertomographie ersetzt. Der Nutzen von Schrägaufnahmen in der Akutdiagnostik wird kontrovers bewertet [7,3]. Spezielle Projektionen wie die transorale Darstellung des Dens axis haben ebenso wenig ihre Bedeutung verloren wie jene zur seitlichen Ablichtung des schwer darstellbaren aber diagnostisch wichtigen zervikothorakalen Überganges: gerade bei kräftigen oder kurzhalsigen Patienten wird die radiologische Darstellung des zervikothorakalen Überganges durch die überlagernde Schulterregion behindert [6]. Durch nach kaudal gezogene Schultern oder in Schwimmeroder Fechterstellung lässt sich diese Region häufig überlagerungsfreier darstellen und bewerten (Abb.2a-c). Gelingt auch hierdurch keine befriedigende Röntgendarstellung und somit nicht die Diagnosestellung oder der Verletzungsausschluss, ist die Indikation zur computertomographischen Abklärung gegeben.

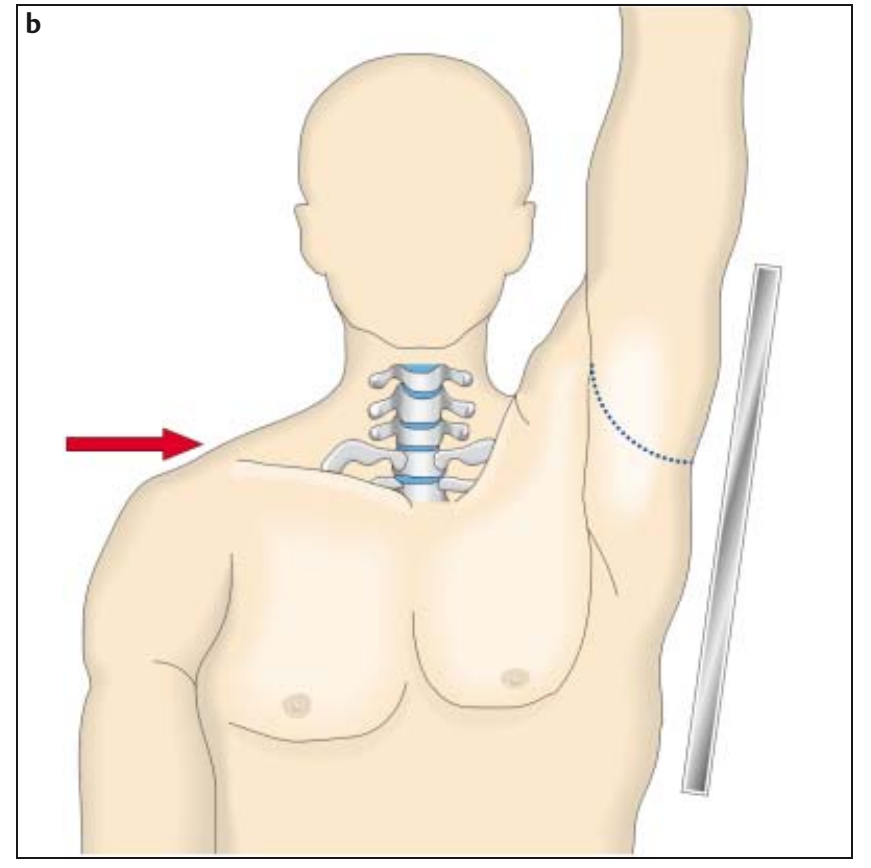

Abb. 2a-c Im seitlichen Strahlengang ist die okzipitozervikale Dissoziation unschwer erkennbar

(a), man beachte jedoch, wie schlecht im Vergleich hierzu die unteren Halswirbelsäulensegmente zur Darstellung kommen. Speziell bei dieser häufigen Problematik helfen Sonderaufnahmen in der sog. Schwimmer- oder Fechterstellung $(\mathbf{b}, \mathbf{c})$ weiter. Lässt sich hierdurch kein suffizientes Ergebnis erzielen, besteht die Indikation zur Durchführung eines CT des zervikothorakalen Überganges.
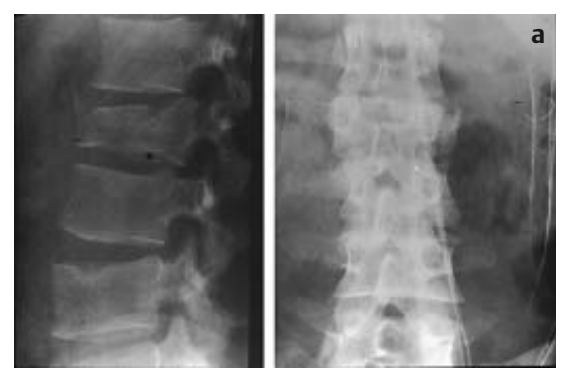

Abb.3a,b Im konventionellen Röntgenbild ist die Fraktur des ersten Lendenwirbelkörpers zwar eindeutig zu diagnostizieren (a), jedoch erst in der Computertomographie werden die erhebliche Verlagerung des Hinterkantenfragmentes nach dorsal sowie zusätzlich der Bogenbruch erkennbar (b).

Funktionsaufnahmen der Halswirbelsäule im seitlichen Strahlengang in Flexion und Extension sind bei geringem logistischen Aufwand unter Kontrolle eines erfahrenen Arztes bei diskoligamentären Verletzungen und segmentaler Instabilität indiziert. Zur raschen Abklärung einer Operationsindikation sind sie in der Akutdiagnostik bei Wirbelsäulenverletzungen der zeit- und kostenaufwendigeren kernspintomographischen Untersuchung vorzuziehen.

Die Standardröntgenaufnahme der Wirbelsäule im antero-posterioren und seitlichen Strahlengang ist der Grundpfeiler der Knochenabbildung.
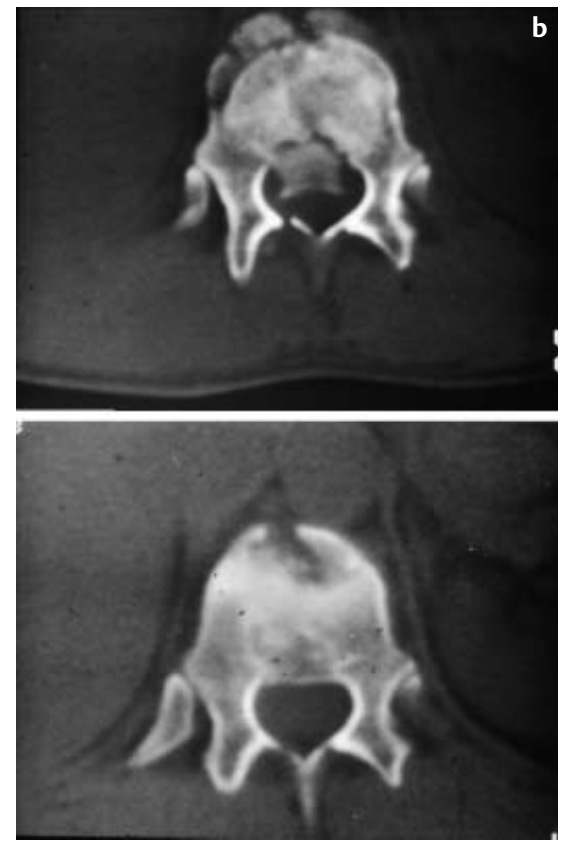

\section{Computertomographie (CT)}

Die computertomographische Untersuchung ist ein radiologisches Verfahren mit einer hohen räumlichen Auflösung und der höchsten Kontrastauflösung im Knochen. Sie gilt heute als Standardtechnik zur Diagnostik von Verletzungen in den Problemregionen der Wirbelsäule und ist ideal zur Bewertung des knöchernen Verletzungsausmaßes, wie Frakturlinienverläufe, Sinterung der Fraktur, Be- 
teiligung der Wirbelkörperhinterkannte als Zeichen der instabilen Fraktur und insbesondere zur Beurteilung der Lagebeziehung von Fragmenten zum Spinalkanal [1] (Abb.3a,b). Gleichzeitig können diskoligamentäre Strukturen und der traumatische Bandscheibenvorfall dargestellt werden.

Mit der Spiral-Computertomographie lassen sich qualitativ hochwertige Sekundärschnittbilder in beliebigen Ebenen, so auch die koronaren Ablichtungen zur Beurteilung des kraniozervikalen Überganges, erstellen. Die früheren Begrenzungen der Computertomographie sind damit überwunden. In der transaxialen Schichtebene verlaufende Frakturen, z.B. nicht dislozierte Densfraktur oder Dislokationen in axialer Richtung und somit senkrecht zur Schichtebene, können nun dargestellt werden $[2,4]$.

Unverzichtbar ist die CT-Untersuchung zur postoperativen Kontrolle des Repositionsergebnisses und bei der Bewertung der Implantatlage nach Frakturstabilisierung (Abb.4au.b).

Die Computertomographie gilt heute als unverzichtbare Untersuchungstechnik zur Beurteilung von Wirbelsäulenverletzungen.

\section{Kernspintomographie (MRT)}

Die Kernspintomographie ist das radiologische Verfahren mit dem höchsten Weichteilkontrast. Besser als die Computertomographie gibt sie somit Auskunft über die diskoligamentären Weichteile und den Inhalt des Spinalkanals (Abb.5). Die knöchernen Strukturen kommen unzureichend zur Darstellung. Für den bewusstlosen oder polytraumatisierten Patienten bedeutet der häufig disloziert stehende Kernspintomograph ein Transportproblem, durch die lange Untersuchungsdauer resultieren Bewegungsartefakte bei eingeschränkter Anästhesiemöglichkeit.

So ist die kernspintomographische Untersuchung lediglich indiziert bei einer $\mathrm{Zu}$ nahme neurologischer Defizite nach Trauma, verursacht durch Bandscheibenvorfall oder intraspinalem Hämatom. Selten handelt es sich jedoch hierbei um akut und isoliert auftretende Pathologika. Häufiger finden sich weitergehende Verletzungsmuster, welche bei der compu-
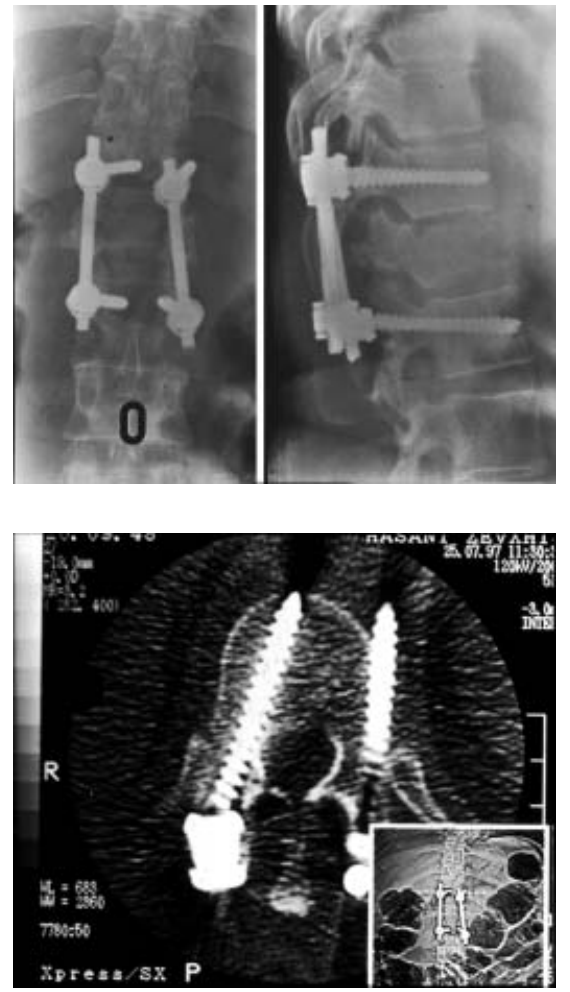

Abb.4a,b Die Computertomographie ist nicht nur bei der präoperativen Diagnostik hilfreich, in diesem speziellen Beispiel muss man bereits anhand des konventionellen Röntgenbildes (a) den Verdacht auf das Vorliegen einer Fehlplatzierung der kranialen linken Schanz-Schraube äußern, was sich in der Computertomographie eindeutig bestätigt (b).

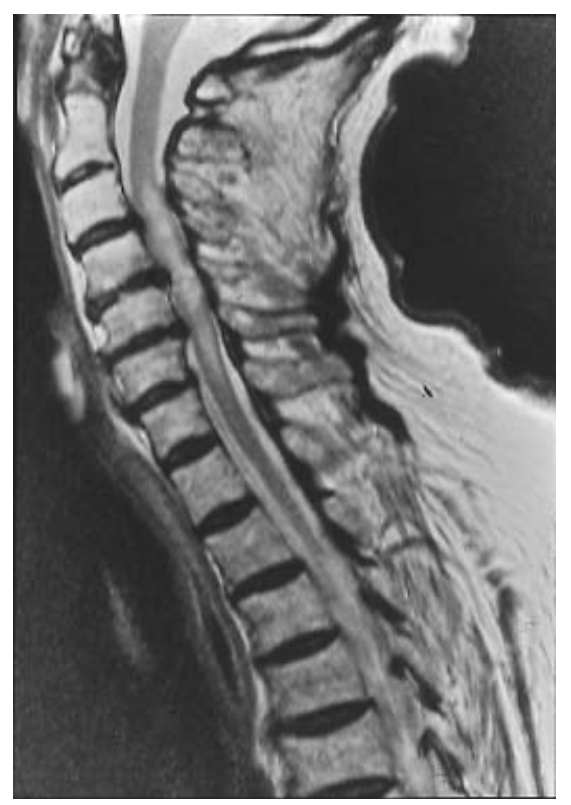

Abb.5 In der MRT-Untersuchung der Halswirbelsäule sind auf Höhe $C 3 / C 4$ deutlich intramedulläre kontusionsbedingte Einblutungen sichtbar. tertomographischen Untersuchung bereits diagnostiziert wurden und die operativen Revision des oder der betroffenen Segmente bereits erforderlich machten. Unmittelbar nach dem Trauma einsetzende neurologische Defizite sind immer Folge direkter Rückenmarkläsionen wie Ödem, Hämatomyelie oder Transsektionen, und stellen keine primäre Indikation für eine kernspintomographische Untersuchung dar [4].

Die Kernspintomographie ist das radiologische Verfahren mit dem höchsten Weichteilkontrast.

Die Indikation für Untersuchungen mit Angio-CT oder Angio-MRT sind besonderen Fragestellungen vorbehalten und gehören nicht in den Bereich der Akutdiagnostik bei akuten Wirbelsäulenverletzungen. Dies gilt auch für die Myelographie, deren frühere Indikationen von der Kernspintomographie übernommen wurden.

\section{Literatur}

Böhmer G, Hierholzer G. Dreidimensionale Rekonstruktionen computertomographischer Wirbelsäulenschichtaufnahmen. OPJournal 1990; 2: 50-53

2 Böhmer G, Roesgen M, Hierholzer G. Dreidimensionale Computertomographie in der Unfallchirurgie. Akt Traumatol 1992; 22 . 47-56

${ }^{3}$ Feemyer B, Knopp R, Piche J, Wales L, Williams J. Comparison of five-view and three-view cervical spine series in the evaluation of patients with cervical trauma. Ann Emerg Med 1989; 18: 818-821

${ }^{4}$ Galinski M, Wippermann B. (Hrsg.) Kompendium der traumatologischen Röntgendiagnostik. Springer Berlin Heidelberg New York 1999

${ }^{5}$ Kortmann H-R, Eggers Ch, Schofer M, Hax PM. Diagnostik und Therapie der Verletzungen der oberen Halswirbelsäule. Trauma Berufskrankh 2000; 2: 134-147

${ }^{6}$ Schneider U, Böhmer G, Hierholzer G. Spezielle Diagnostik bei Wirbelsäulenverletzungen. OP-Journal 1996; 2: 150-154

Zöllner G, Dietemann JL, Dosch JCl. Radiologische Frühbeurteilung der Wirbelverletzung. Z Orthop 1992; 130: 435-440

\section{Dr. med. Georg Böhmer Oberarzt Dr. med. Hermann-Josef Böhm Oberarzt}

Berufsgenossenschaftliche Unfallklinik Großenbaumer Allee 250 D-47249 Duisburg 